\title{
GSPROJECTS - AMBIENTE PARA SIMULAÇÃO DA GESTÃO DE PROJETOS DE SOFTWARE
}

\author{
Danilo Rangel Arruda Leite ${ }^{1}$, Samyr Santos Delfino ${ }^{1}$, Cláudia Batista Mélo , \\ Álvaro Francisco Medeiros ${ }^{2}$, Hálamo Giulian Reis de Andrade ${ }^{2}$ \\ ${ }^{1}$ Setor de Gestão de Processos e Tecnologia da Informação (SGPTI) - Empresa \\ Brasileira de Serviços Hospitalares (EBSERH) - Hospital Universitário Lauro \\ Wanderley (HULW) - Universidade Federal da Paraíba (UFPB) \\ João Pessoa - PB - Brasil \\ ${ }^{2}$ Centro de Informática - Universidade Federal da Paraíba (UFPB) \\ João Pessoa - PB - Brasil \\ danilorangel@buscapb.com.br, \{afcmedeiros, claudiabmelo, samyrsd, \\ halamoreis\}@gmail.com
}

\begin{abstract}
The use of games for education and training has a high power of attraction compared to other forms of learning. Featured games for this purpose are classified as serious games, as they associate specific content to play activities providing engagement and significant results when properly modeled. This article presents the proposal of GSPROJECTS, a serious games aimed at the sustainable management of projects using real-world scenarios in which students of the Information Technology are causing to use their knowledge in driving and problem solving within a project. The GSPROJECTS stimulates cognitive functions associated with the perception and learning, allowing the teacher to edit the challenges through the questionnaire module, and the game to adapt to different curricula of undergraduate courses in Computer and Information Technology area.
\end{abstract}

Resumo. A utilização de jogos para educação e treinamento tem um alto poder de atração se comparado a outras formas de aprendizado. Jogos caracterizados por esta finalidade são classificados como jogos sérios, pois associam conteúdos específicos a aspectos lúdicos, proporcionando um engajamento e resultados significantes quando bem modelados. Este artigo traz a proposta do GSPROJECTS, um jogo sério voltado para a gestão de projetos sustentável utilizando cenários reais, no qual alunos da área de Tecnologia da Informação são provocados a utilizar os seus conhecimentos na condução e resolução de problemas dentro de um projeto. $O$ GSPROJECTS estimula as funções cognitivas, associadas à percepção e à aprendizagem, permitindo ao professor editar os desafios por meio do módulo questionário, possibilitando que o jogo se adapte aos diferentes currículos dos cursos de graduação na área de Computação e Informática.

Palavras-chave: Gerenciamento de projetos, Simulação, Ensino, jogos sérios 


\section{Introdução}

A aplicação de técnicas de gerenciamento em projetos de software, muitas vezes, é ineficiente e inadequada, podendo influenciar nos fracassos dos projetos. O alto número de projetos que, a cada ano, apresentam problemas de cronograma, orçamento e, em muitos casos, chegando ao seu cancelamento, pode ser consequência desta deficiência [Schoeffel 2014]. Uma das possíveis causas destas falhas em gerenciamento é a falta de um melhor entendimento das suas práticas e técnicas essenciais para gerenciar projetos.

A utilização de jogos na educação proporciona aos estudantes e profissionais um ambiente interativo e dinâmico, provocando a motivação para avançar em um enredo, por meio de simulações e situações relacionadas ao tema abordado. A resolução de problemas nestes ambientes está fortemente associada às funções cognitivas [Bittencourt e Giraffa 2003].

Jogos com apelo educacional possuem uma maior interação com seus usuários, além disso, este tipo de aplicação torna o aprendizado mais atrativo. Assim, a utilização de jogos mostra-se como uma excelente alternativa no processo ensino aprendizagem, principalmente, quando o ambiente que o jogo foi produzido não é de fácil acesso ou existem riscos em caso de erro.

No cenário da gestão de projetos de software, por exemplo, a imersão de alunos e profissionais em um ambiente real é mais complexo, pois a condução de um projeto normalmente está associado a metodologias e processos, podendo destacar o Project Management Body of Knowledge (PMBOK), Information Technology Infrastructure Library (ITIL) e Control Objectives for Information and relatet Technology (COBIT), que envolvem pessoas e custos. Simular um ambiente deste na vida real é mais complexo, levando-se em consideração que um projeto de software pode ser conduzido de formas diferentes e seguir variados caminhos e decisões que são divergentes entre si e ainda assim ser concluído com sucesso.

Atualmente, existem vários jogos com esse propósito como, por exemplo, o TIM: The Incredible Manager [Dantas et al. 2004], o SimSE [Navarro et al. 2004] e o SESAM [Drappa e Ludewig 2007]. Através de uma revisão da literatura, buscou-se auxílio para identificar características desses jogos que possam ajudar a formação das competências dos gerentes de projeto de software.

Dentro deste contexto, este artigo tem como objetivo descrever a proposta do GSPROJECTS (Games for Simulation Projects), um jogo sério para gestão de projetos de software. Este jogo de simulação busca suprir algumas das necessidades observadas em Paludo e colaboradores (2010), principalmente, para alunos iniciantes na área de Tecnologia da Informação (TI).

O simulador proposto apresenta algumas semelhanças com os jogos de empresa utilizados na área de Administração ${ }^{1}$. Jogos de empresa são simulações de um ambiente empresarial, onde os participantes atuam como executivos de uma empresa avaliando e analisando cenários hipotéticos e suas possíveis consequências.

\footnotetext{
1 O Desafio Sebrae é um jogo virtual que simula o dia-a-dia de uma empresa, durante mais de seis meses, organizados em equipes, universitários de todo o país, testam sua capacidade de administrar um negócio, tomar decisões e trabalhar em equipe.
} 
O jogo foca no gerenciamento de escopo, onde grande parte dos problemas é decorrente da falta de planejamento e controle do escopo e, segundo PMI (2014), possue o processo de criação da Estrutura Analítica do Projeto (EAP). A EAP deve ser dominada, visando ao adequado gerenciamento de um projeto e é base para uma grande quantidade de outros processos que poderão ser utilizados em futuros módulos do simulador [PMI 2014].

\section{Gerenciamento do escopo de projeto de software}

Em sua quinta edição, o PMBOK apresenta as melhores práticas para gerenciamento de projetos, contendo uma descrição sobre o conjunto de processos necessários para realizar a atividade de gerenciamento de projetos. De acordo com o PMI (2014), "projeto é um esforço temporário empreendido para criar um produto, serviço ou resultado exclusivo". Neste sentido, ainda segundo o PMI (2014), a atividade de gerenciamento de projetos é a "aplicação de conhecimentos, habilidades, ferramentas e técnicas às atividades do projeto a fim de atender a seus requisitos".

A atividade de gerenciamento de projeto vem crescendo muito ao longo do tempo, sendo que a área de TI se destaca como sendo uma das áreas que mais utilizam as técnicas de gerenciamento de projetos na condução dos seus projetos, segundo pesquisa realizada pelo Project Management Institute (PMI). De acordo com a pesquisa de benchmarking realizada pelo PMI, em 2010, com todas as suas unidades do Brasil, a área de TI apresenta 64,8\% de utilização das técnicas de gerenciamento de projetos, sendo seguida pela área de engenharia com $44,8 \%$. Ainda de acordo com esta pesquisa, a área de escopo se destaca como sendo o segundo aspecto mais importante, com $96,5 \%$, perdendo apenas para o tempo com $98,4 \%$.

O Gerenciamento de Projetos de Software utiliza conhecimentos, habilidades, ferramentas e técnicas nas atividades do projeto, a fim de atender aos seus requisitos. $\mathrm{O}$ gerente de projetos é a pessoa responsável pela realização dos objetivos do projeto. Conhecer e aplicar ferramentas e técnicas reconhecidas como boas práticas não são suficientes isoladamente para um gerenciamento de projetos eficaz.

De acordo com o PMBOK, é necessário que a equipe de gerenciamento de projetos entenda e use o conhecimento e as habilidades de pelo menos cinco áreas de especialização [PMI 2014]: o conjunto de conhecimentos em gerenciamento de projetos; conhecimento, normas e regulamentos da área de aplicação; entendimento do ambiente do projeto; conhecimento e habilidades de gerenciamento geral; e habilidades interpessoais.

Carbone e Gholston (2004) afirmam que o papel do gerente é vital para o projeto e para o seu desenvolvimento, ou seja, a melhoria dos resultados dos projetos da organização necessita do desenvolvimento das competências de seus gerentes. Neste contexto, métodos de apoio ao ensino de gerência de projetos de software que possibilitem o estudante a vivenciar situações próximas às reais, onde aprendem fazendo, precisam ser incorporados ao treinamento tradicional. Neste caso, acredita-se que os jogos educativos por computador podem contribuir diretamente. 


\section{Jogos Sérios}

Segundo Battistela e colaboradores (2014), a aprendizagem baseada em jogos desenvolve nos alunos uma aprendizagem ativa, permitindo, em alguns casos, uma maior participação e compreensão do conteúdo.

A utilização de jogos para treinamento e simulação está presente na literatura há vários anos, dentre estes jogos existe uma categoria que vem sendo definida como jogos sérios. Tais jogos não possuem como único objetivo o entretenimento, pois incluem características voltadas para o ensino, formação e informação [Michael e Chen 2005]. Entretanto, segundo Zyda (2005) e Machado e colaboradores (2011) esta definição ainda não é exata, mas é associada a uma categoria de jogos que tem como objetivo simular situações reais, permitindo aos profissionais a oportunidade de experimentar situações críticas e consolidar seus conhecimentos e habilidades por meio da resolução de problemas em assuntos específicos.

Para que estes jogos sejam atraentes e realmente provoquem nos seus usuários instigação e tempestividade no engajamento para resolução de problemas, com embasamento teórico e prático, sem tornar-se monótono, geralmente, são utilizadas na sua elaboração conhecidas técnicas e estratégias já consolidadas na indústria dos jogos de entretenimento. Dessa forma, tais simulações proporcionam tarefas que estimulam a construção de teorias e funções psicomotoras [Machado et al. 2011].

Tais jogos são utilizados para treinamento e educação em variados seguimentos como, por exemplo, na área de Saúde podem ser citados os trabalhos de Machado (2009) e Johnsen e colaboradores (2007); na Engenharia podem ser citados os trabalhos de Morsi e Jackson (2007) e Chagas e colaboradores (2012); e na Ciência da Computação pode ser citado o trabalho de Hakulinen (2011). A utilização e obtenção de resultados significativos validam as pesquisas na implementação deste tipo de ferramenta como apoio ao aprendizado e especialização das habilidades cognitivas.

Quando planejados e implementados corretamente, tais jogos podem utilizar a excitação demonstrada pelos jogadores a seu favor, de forma que as habilidades de utilidade prática e as competências transferidas possam ser aplicadas no mundo real, sendo este o principal diferencial dos jogos sérios [Greitzer et al. 2007, Vorderer et al. 2003].

\section{GSPROJECTS}

O GSPROJECTS, Games for Simulation Projects, é um jogo sério para gestão de projetos de software. Para elaboração da primeira versão do GSPROJECTS, foram utilizados projetos reais com documentação e registros informando todos os procedimentos e problemas que foram encontrados na condução do mesmo.

As principais características dos simuladores como interatividade, solução de problemas, feedback multimídia e imitação da vida real [Akilli 2007] parecem adequadas para uma melhor motivação e engajamento dos estudantes, permitindo maior facilidade de utilização e interpretação durante a execução de simulações. 
O GSPROJECTS tem por finalidade apoiar o ensino de conceitos de gerência de projetos [Prikladnicki et al. 2007], seguindo algumas orientações de Paludo e colaboradores (2010), tais como:

- Modelar o perfil dos personagens do jogo: considera características como personalidade e perfil de liderança do jogador que será o gerente do projeto e características como personalidade, afinidade, habilidade, custo, experiência e produtividade para os membros da equipe do projeto;

- Estar disponível na plataforma Web: o acesso em qualquer lugar e hora, sem complicações com configurações, é um facilitador importante;

- Emitir relatórios e análises gráficas das ações do jogador: como forma de reforço pedagógico, indicando possíveis equívocos e estatísticos coletados durante o jogo como má alocação de pessoas ou distribuição de tarefas inadequadas.

Vale ressaltar que, por se tratar de uma aplicação com objetivos específicos, é essencial o envolvimento de profissionais da área para a qual o jogo está sendo planejado ou se relaciona [Machado et al. 2011].

No refinamento do GSPROJECTS será utilizada a abordagem implementada no trabalho de Linehan e colaboradores (2009) na qual foram convidados diversos profissionais da área e os mesmos foram provocados a tomar decisões em um jogo implementado em papel, servindo estas entradas e o feedback dos mesmos como balizadores para realização de melhorias e ajustes antes das integrações de desenvolvimento do jogo.

A primeira versão do GSPROJECTS não simula todos os processos utilizados na gerência de projetos, mas tem como foco o grupo de processos de planejamento. Inicialemente, foi abordado o gerenciamento de escopo do projeto.

O GSPROJECTS é atrativo para os estudantes, possibilitando que eles testem suas habilidades na gestão do desenvolvimento de software. O jogo conta com um módulo de administração no qual diversos professores podem gerenciar a evolução de seus alunos. A cada finalização de etapa do jogo, é dado um feedback ao estudante para que ele fique sabendo onde errou, permitindo retornar à fase anterior e rever suas escolhas. Cada fase representa um modelo de simulação distinto, configurado no ambiente administrado pelo professor, oferecendo assim uma flexibilidade que permite a adoção de diferentes metas educacionais, seja em nível profissional, graduação ou de pós-graduação, utilizando o mesmo jogo com diferentes modelos.

$\mathrm{O}$ jogador atua como gerente de projetos em um ambiente de desenvolvimento de software no qual sua equipe executa várias tarefas com um cronograma planejado.

Os personagens do GSPROJECTS são:

- Gerente - papel assumido pelo jogador que recebe a descrição do projeto no início da fase e é responsável pelo seu planejamento e execução, vivenciando diversas situações de tomada de decisões ao longo do desenvolvimento do projeto de forma a garantir que ele será completado dentro das estimativas previstas;

- Profissionais - personagens que compõem a equipe de desenvolvimento com que o gerente vai contratar e contar para o projeto; 
- Patrocinador (Professor) - personagem a quem o gerente deve obedecer. O patrocinador é quem faz a aceitação do plano de projeto, do cronograma e custos propostos pelo gerente.

O jogador deve passar por várias etapas que descrevem o início do projeto: a criação de um plano de projeto, sua aceitação pelos patrocinadores (professor), a execução e o controle da rede de tarefas até que o projeto chegue ao seu fim. Estas etapas estão ilustradas na Figura 1 e são apresentadas em detalhes a seguir.

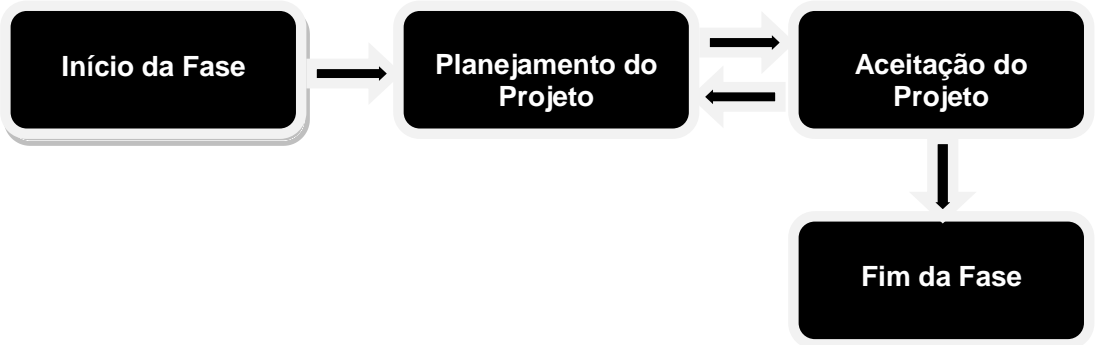

Figura 1: Etapas de uma fase do jogo

A utilização de múltiplas fases permite que várias situações e políticas gerenciais possam ser apresentadas ao jogador, sequencialmente, em diferentes configurações de projetos de software, tornando o jogo um instrumento mais flexível para treinamentos.

Ao iniciar o jogo, é apresentada ao jogador uma tela com as opções "Novo Jogo", "Como Jogar" e "Estatísticas". Ao clicar em "Novo Jogo", inicia uma nova partida. Clicando em "Como jogar", o jogador tem acesso a um pequeno tutorial contendo as instruções necessárias ao entendimento do jogo. Clicando em estatísticas, é listado um ranking com as dez melhores pontuações do jogo.

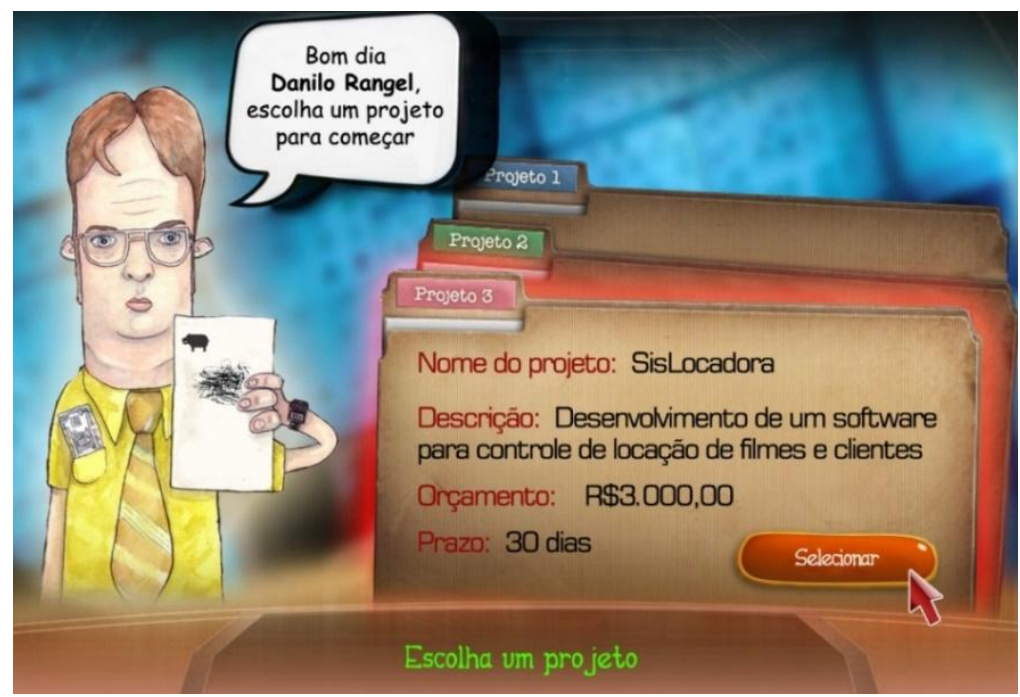

Figura 2: Escolha do projeto 
A Figura 2 apresenta uma tela onde o jogador precisa escolher entre vários projetos disponíveis. Cada projeto pode ser considerado como processo de inicialização, onde é desenvolvido o termo de abertura do projeto ${ }^{2}$.

Além da escolha do projeto, as demais fases do jogo são:

- Planejamento do Projeto - nesta etapa, o jogador deverá criar o plano do projeto que será executado durante o desenvolvimento do produto, criação do Escopo e EAP;

- Criação do Escopo do Projeto - o escopo inclui os processos necessários para garantir que o projeto possua todo o trabalho necessário, e somente ele, para terminar o projeto com sucesso [PMI 2014];

- EAP - nesta fase, o jogador exercita o conceito de estrutura analítica do projeto de forma prática;

- Definir Equipe - a definição da equipe consiste em alocar um desenvolvedor para cada pacote de trabalho, sendo que não pode haver mais de um desenvolvedor alocado em uma mesma tarefa;

- Definição de Atividades - uma vez escolhida à equipe, a próxima etapa será atribuir os profissionais as atividades que serão desenvolvidas;

- Aceitação do Projeto - o plano criado pelo jogador poderá ser aceito ou recusado pelo patrocinador do projeto (professor);

- Fim da Fase - ao finalizar o jogo, o jogador é direcionado para um questionário de avaliação do experimento. Através dele será possível fazer uma análise quantitativa da eficácia do jogo nas práticas de gerência de projeto.

\section{Avaliação do GSPROJECTS}

Após desenvolvimento da primeira versão, o GSPROJECTS foi aplicado de forma experimental, como atividade prática, possibilitando a validação dessa proposta. Este experimento contou com a participação de 20 alunos divididos em dois grupos de 10 alunos cada (Grupo 1 e Grupo 2). O objetivo principal foi verificar como o GSPROJECTS poderia auxiliar no aprendizado de Gerenciamento de Projetos.

Foram escolhidos estudantes matriculados na disciplina de Gerenciamento de Projetos, do $5^{\circ}$ período, do Curso de Ciência da Computação, de uma instituição de ensino superior privado da cidade de João Pessoa, no estado da Paraíba.

Os testes ocorreram em quatro dias, sendo ministradas duas aulas teóricas(primeiro e segundo dia) sobre conceitos de Gerenciamento de Projetos e Escopo.

No terceiro e quarto dias foram aplicados exercícios práticos com a turma dividida em dois grupos: comparação e controle. O grupo controle exercitava o conteúdo aprendido na sala de aula com a utilização do GSPROJECTS, enquanto o grupo de comparação respondia os exercícios de forma tradicional.

\footnotetext{
${ }^{2}$ É a autorização para o início do projeto ou de uma de suas fases, documentando as suas necessidades e
} conectando o projeto aos trabalhos em andamento da organização [Valle et al, 2010]. 
No decorrer do experimento, cada grupo teve a oportunidade de ser tanto de comparação quanto de controle. Isso evitou que desvios nos resultados ocorressem devido a diferenças individuais de conhecimento dos estudantes de cada grupo.

Ao final de cada aula, foi aplicada uma prova de cinco questões, contemplando o conteúdo ministrado. Essa prova teve a função de avaliar o nível de entendimento do conteúdo tanto no grupo que teve a simulação com a utilização do GSPROJECTS, quanto no grupo que a aula foi ministrada através do método tradicional de ensino.

Realizando uma comparação entre os resultados obtidos em uma mesma turma nos dois dias de testes, percebeu-se que quando o grupo 1 foi o grupo controle, ou seja, utilizou o jogo, sua média geral de acertos foi de $80 \%$, bem superior aos $40 \%$ de acertos obtidos quando este mesmo grupo foi o grupo comparação.

Estes valores já poderiam demonstrar um ganho de desempenho entre os estudantes de uma mesma turma nos testes feitos durante as aulas com a utilização do GSPROJECTS. Os alunos relataram que, com a utilização do jogo, o interesse e o entendimento melhoraram significativamente.

Para corroborar com essa avaliação, foi realizada uma comparação entre os grupos, no mesmo dia de aula. $\mathrm{O}$ grupo que fez os exercícios práticos utilizando o jogo obteve uma pontuação superior ao grupo que não utilizou. Como exemplo, podemos tomar os resultados obtidos no primeiro dia do estudo. Neste dia, o grupo 1, foi o grupo controle, obteve uma média de acertos de $80 \%$ de questões corretas, enquanto que o grupo 2, grupo comparação, obteve uma média de acertos de $50 \%$ de questões corretas, indicando uma tendência de crescimento no desempenho das turmas com a utilização do GSPROJECTS. No segundo dia do estudo, o grupo 1, foi o grupo comparação, obteve uma média de $40 \%$ de questões corretas, enquanto o grupo 2 , grupo controle, obteve uma média de $75 \%$ de questões corretas. Estes valores demonstram um ganho de desempenho entre os estudantes de uma mesma turma nos testes feitos durante as aulas com a utilização do GSPROJECTS.

O treinamento não foi considerado cansativo. Os estudantes afirmaram que a experiência com o GSPROJECTS foi estimulante, dinâmica, prática e proveitosa. A interface foi considerada atrativa e amigável ao usuário, fazendo com que o tempo passasse despercebido durante o uso desse jogo.

Dentre as vantagens relatadas pelos usuários do GSPROJECTS, podem ser destacadas: interface amigável, fácil utilização do jogo, plataforma Web, possibilidade de simular em vários cenários criados pelo professor, rápido feedback, módulo para o professor criar e avaliar os projetos finalizados.

\section{Comentários Finais}

A utilização de jogos de simulação alinhados às formas tradicionais de ensino (livrostexto e aulas expositivas) proporciona benefícios como motivação e engajamento dos estudantes durante o treinamento, contribuindo assim para sua melhor eficiência.

Este trabalho apresentou o GSPROJECTS, uma ferramenta computacional para auxiliar a formação de gerentes de projetos de software, de forma lúdica e agradável. Isto pode ser um fator importante na diminuição dos problemas e fracassos comuns aos 
projetos de software, treinamento e entretenimento, unindo dois grandes seguimentos do mercado.

O jogo sério proposto neste trabalho encontra-se em fase de refinamento do enredo e testes de validação. Vale ressaltar as principais características do GSPROJECTS:

- Ser de fácil utilização: possui interface autoexplicativa para facilitar sua aceitação em sala de aula como material de apoio ao ensino;

- Possuir diversidade de recursos pedagógicos: indica bibliografia para reforçar estudos, links para informações adicionais de conteúdos e manual de instruções;

- Oferecer funcionalidades para múltiplos jogadores;

- Possibilitar a criação de cenários pelo usuário ou professor;

- Enfatizar a motivação, engajamento, recompensas, desafios e efeitos visuais: procurando despertar o interesse e a curiosidade dos alunos;

Como trabalhos futuros, poderão ser feitas melhorias e otimizações no GSPROJECTS, bem como, poderão ser adicionadas outras fases da gestão de projetos.

\section{Referência}

Battistela, Paulo E, Wangenheim, Christiane G. Von, Fernandes, João M (2014): Como jogos educacionais são desenvolvidos? Uma revisão sistemática da literatura, WEI CSBC 2014 - http://hdl.handle.net/1822/33145.

Bittencourt, J.R., Giraffa, L.M.M. (2003) "Modelando Ambientes de Aprendizagem Virtuais utilizando Role-Playing Games". XIV Simpósio Brasileiro de Informática na Educação - NCE - IM/UFRJ.

Carbone, T. A., Gholston, S. (2004) Project Manager Skill Development. "A Survey of Programs and Practitioners. Engineering Management" Journal Vol. 16 No. 3 September.

Chagas, J. F. S., Neto, F. M. M., Brasil, I. S., Monteiro, R., Bonates, M. F. (2012) "Um Jogo Para Treinamento De Operadores De Sondas De Perfuração Terrestre". HOLOS, Ano 28, Vol 1. ISSN 1807 - 1600.

Dantas, A.A., Barros, M.O., Werner, C.M.L. (2004) “ Treinamento Experimental com Jogos de Simulação para Gerentes de Projeto de Software." Laboratório de Engenharia de Software. Programa de Engenharia de Sistemas e Computação. COPPE/UFRJ.

Greitzer, F.L., Kuchar, O.A., Huston, K. (2007) "Cognitive science implications for enhancing training effectiveness in a serious gaming context." ACM Journal of Educational Resources in Computing, 7, 3, Article 2.

Hakulinen, L. (2011) "Using serious games in computer science education". In Proceedings of the 11th Koli Calling International Conference on Computing Education Research (Koli Calling '11). ACM, New York, NY, USA, 83-88. DOI=10.1145/2094131.2094147 http://doi.acm.org/10.1145/2094131.2094147 
Johnsen, K., Raij, A., Stevens, A., Lind, D. S., Lok,B. (2007) "The validity of a virtual human experience for interpersonal skills education". In Proceedings of the SIGCHI conference on Human factors in computing systems (CHI '07). ACM, New York, NY, USA, 1049-1058. DOI=10.1145/1240624.1240784 http://doi.acm.org/10.1145/1240624.1240784.

Linehan, C., Lawson, S., Doughty, M., Kirman, B. (2009). "Developing a serious games to evaluate and train group decision making skills". In Proceedings of the 13th International MindTrek Conference: Everyday Life in the Ubiquitous Era (MindTrek '09). ACM, New York, NY, USA, 106-113. DOI=10.1145/1621841.1621861 http://doi.acm.org/10.1145/1621841.1621861.

Machado, L. S., Moraes, R. M. (2009) "Qualitative and Quantitative Assessment for a VR-Based Simulator". Studies Health Technol Informatics;142:168-73.

Machado, L. S., Moraes, R. M., Nunes, F. L. S., Costa, R. M. E. M. (2011) "Jogos sérios Baseados em Realidade Virtual para Educação Médica". Revista Brasileira De Educação Médica: 254 - 262 ;

Michael, D. R., Chen, S. L. (2005)." Serious games: Games that Educate, Train, and Inform". Muska \& Lipman/Premier-Trade.

Morsi, R., Jackson, E. (2007) "Playing and learning? educational gaming for engineering education" . In Frontiers In Education Conference - Global Engineering: Knowledge Without Borders, Opportunities Without Passports, 2007. FIE '07. 37th Annual, pages F2H-1-F2H-6.

Navarro, E.,Baker, A., Hoek. (2004) SIMSE: An interactive simulation game for softtware engineering education. Information and Computer Science University of California.

Paludo L., Raabe A. (2010) "Análise de Jogos Educativos de Computador para Gerência de Projetos de Software". XXX Congresso da SBC. Belo Horizonte.

PMI (2014) "Um Guia do Conhecimento em Gerenciamento de Projetos (Guia PMBOK $\left.{ }^{\circledR}\right) ” .5^{a}$ Ed. Project Management Institute.

Schoeffel, Pablo, (2014) PizzaMia: Dinâmica Vivencial para Apoio ao Ensino de Gerenciamento de Projetos Baseado no PMBOK, WEI - CSBC 2014 - Universidade de Brasília.

Vorderer, P., Hartmann, T., Klimmt, C. (2003) "Explaining the enjoyment of playing video games: the role of competition," in Proceedings of the second international conference on Entertainment computing, 1-9, Pittsburgh, PA, USA: Carnegie Mellon University. 